Title: The influence of steering wheel size when tuning power assistance

Journal: Int. J. of Heavy Vehicle Systems, 2014 Vol.21, No.4, pp.295 - 309

DOI: $10.1504 /$ IJHVS.2014.068099

Copyright: (C) 2014 Inderscience Enterprises Ltd.

Version: Author's post-peer review

To cite this article: TAGESSON, Kristoffer; JACOBSON, Bengt; LAINE, Leo. The influence of steering wheel size when tuning power assistance. International Journal of Heavy Vehicle Systems, 2014, 21.4: 295-309.

To link to this article: http://dx.doi.org/10.1504/IJHVS.2014.068099 


\title{
The Influence of Steering Wheel Size when Tuning Power Assistance
}

\begin{abstract}
This paper describes how steering assistance should scale with steering wheel size. A method has been developed to scale complete torque felt by the driver, both for continuous and discontinuous feedback. This was used in an experiment with 17 subjects all driving a truck with three differently sized steering wheels. The test took place on a handling track at $45 \mathrm{~km} / \mathrm{h}$ to $90 \mathrm{~km} / \mathrm{h}$. Continuous feedback was evaluated subjectively; discontinuous feedback by measuring angular response. Results show that torque feedback should decrease as steering wheel size decreases. A rule of thumb is to keep driver force level constant to maintain perceived handling and comfort. This also maintained the average steering wheel angle change response to discontinuous assistance. Furthermore large variance in angular response was observed. The direction, measured 0.25 $\mathrm{s}$ after start of a pulse, was the same as that of the pulse applied in $88 \%$ of the recordings.
\end{abstract}

Keywords: steering wheel size; tuning; power assistance; steering assistance; torque feedback; force feedback; trucks; angular response; handling; comfort; heavy vehicles; steering wheel diameter; steering wheel radius; steering haptics

\section{Introduction}

Power steering, also known as power assistance, has been used for many years to lower the required steering effort when turning a vehicle or a vessel (Howe, 1956). In recent years various more advanced steering systems have emerged. These include new refined methods for applying assistance torque and changing steering ratio (Heissing and Ersoy, 2010). Also, they are most often electronically controlled which allows for a greater flexibility in behaviour. It also opens up for precise tuning of steering support level at every speed and driving situation.

The steering wheel size, i.e. the diameter, in cars has been kept rather constant historically. Today it is 35-40 cm in most passenger cars (Wheelskins-Inc, 2009). In heavy trucks it is larger as a consequence of legislation. As stated by UNECE (2005) the driver should be able to manoeuvre with limited steering forces also in the case of an assistance failure. With common wheelbase and steering ratio this typically means a steering wheel, $\mathrm{StW}$, diameter of $45-50 \mathrm{~cm}$ on modern heavy trucks. Until recently truck power steering systems have exclusively been hydraulic (Volvo-Trucks, 2013; ZF-Lenksysteme, 2012). When moving away from pure hydraulic power assistance systems and introducing new redundant electronic steering systems the legislation requirement becomes less relevant. Hence, the StW size could be chosen more freely also in a heavy truck.

The importance of force feedback for vehicle handling has been analysed in several studies (Ciarla et al., 2012; Kim and Cole, 2011; Pfeffer, 2006). Anand et al. (2011) carried out a simulator study where steering effort was tuneable when driving. The subjects reported their preferred level when satisfied. It was shown that the level varied highly between 
individuals; this is also confirmed by Barthenheier and Winner (2003). Newberry et al. (2007) performed a thorough experiment to analyse what aspect of force feedback that a driver senses - whether it is torque or force. An indoor test apparatus with a strain gauge was used to measure $\mathrm{StW}$ torque. The $\mathrm{StW}$ angular degree of freedom was locked (isometric test). It was concluded that a driver perceives force rather than torque. When the angular degree of freedom was unlocked and torque set to zero it was further found that a driver perceives StW angle rather than hand translation. The StW centre position was not adjusted when changing StW.

It has not been shown how force feedback should vary with StW size in a real vehicle where isometric motion no longer holds and where the driver subjectively decides on optimal balance between handling and comfort. Also no such test has been performed where the subjects are free to choose seating and StW position which could be different when changing $\mathrm{StW}$. This is the first identified gap analysed in this paper.

Now, continuing on next topic. In recent years new functions have been developed where more than vehicle forces can be sensed in the StW. Examples are functions for positioning in lane, crash avoidance and vehicle stabilisation (Rossetter, 2003; Volvo Cars Corporation, 2008; Yang, 2013). Some of these are not continuous, meaning that the torque which is added to the steering column is ramped up almost instantaneously. The intention is not only to provide tactile information to the driver. It is also to directly effect the steering wheel angle before the driver has had time to understand the seriousness of the situation. During that time the driver is however still in contact with the StW. This highly effects the angular response of the additional torque.

Cole (2012) developed a driver-vehicle model which was used to analyse a driver's ability to do path-following when exposed to a lateral force disturbance. Also driver response from both angle and torque disturbance inputs was analysed. The model was validated with a driving simulator experiment where angle overlay was performed. Groups were divided as tensed or not tensed. Abbink et al. (2011) measured driver frequency response to torque disturbance in a fixed based simulator. Pick and Cole (2007) used a similar set up to validate a linear mass-damper-spring model. The angular response was shown to be captured up to $6 \mathrm{~Hz}$ for random torque disturbances. The model contained arm inertia, damping and stiffness. It was observed that damping and stiffness increased as the driver co-contracted.

Several models have been developed and tests have been performed to study driver response to discontinuous StW torque feedback. No in vehicle measurements have been performed to show differences of driver response when StW size is changed and when the tension level of the driver is a consequence of the main mission of following a real road, or similar. This is the second identified gap analysed in this paper.

When changing StW size old knowledge about truck steering tuning would have to be transferred to apply for another StW size. I.e. how steering assistance should be tuned as a function of $\mathrm{StW}$ size. Considered are both continuous properties, e.g. normal driving characteristics, and discontinuous, e.g. guiding pulse. Hereafter referred to as part 1 and 2 respectively. The work also intends to serve as a mapping when comparing results received from vehicles with different $\mathrm{StW}$ sizes.

This paper describes an experiment conducted on a test track to study:

Part 1 - Tuning of Continuous Characteristics: How continuous steering properties should depend on StW size to maintain a subjective balance between handling and comfort.

Part 2 - Discontinuous Column Torque: How discontinuous steering properties should depend on StW size to maintain the same angular driver response. 
Similar questions could be stated for the properties steering gear ratio and wheelbase. These are not considered and therefore kept fixed.

In the next section of the paper the developed method for scaling is presented and the performed experiment is described. In Section 3 received results are presented and discussed. A conclusion is given in Section 4.

\section{Method}

In this section basics about steering characteristics are described and also how a scaling method was applied. The chosen pulse for discontinuous testing is then described. Finally used vehicle, track and experimental set up are shown.

\subsection{Part 1: Continuous Steering Characteristics}

Steering assistance systems together with vehicle geometries make up for the basic steering characteristic, which is of high importance for the experienced stability of the vehicle. Figure 1 show the basic characteristic properties at $v_{x}=80 \mathrm{~km} / \mathrm{h}$, high friction and low steering wheel angular rate for the truck used in the experiment. A hysteresis, such as seen in Figure 1, appears due to friction and damping in the steering system. On how to measure the characteristics, see e.g. (Salaani et al., 2004). The slope and level of hysteresis of this curve is of high importance to get a comfortable and directionally stable vehicle (Rothhämel, 2010). The term steering characteristics hereafter refer to the relation between $\mathrm{StW}$ torque and $\mathrm{StW}$ angle (with time derivatives). When changing steering wheel diameter

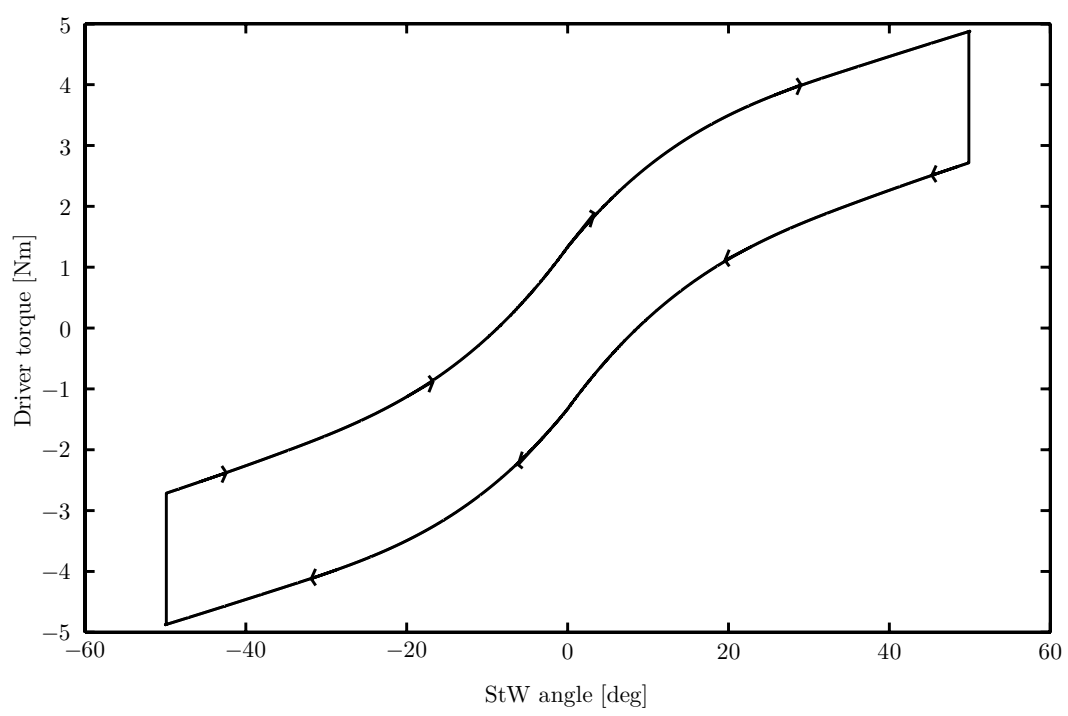

Figure 1 Baseline StW torque characteristics at speed $v_{x}=80 \mathrm{~km} / \mathrm{h}$ for used truck.

the lever arm for the driver will change. One natural hypothesis would be that the drivers force level should be kept constant, when steering angular ratio is maintained. In other 
words the required steering torque should be scaled linearly with steering wheel radius. In this experiment a truck was equipped with Volvo Dynamic Steering (Volvo-Trucks, 2013). This allows for great flexibility in shaping the required steering torque. The torque was changed with a gain factor according to

$$
T_{d}\left(\theta, \dot{\theta}, v_{x}\right)=k_{g} \cdot T_{d, 0}\left(\theta, \dot{\theta}, v_{x}\right)
$$

where $T_{d}$ denotes required driver torque, $\theta$ steering wheel angle, $\dot{\theta}$ steering wheel angular rate, $v_{x}$ vehicle longitudinal speed, $T_{d, 0}$ baseline steering torque characteristics (as in Figure 1 ) and $k_{g}$ a scaling parameter. In Figure 2 characteristics for $k_{g}=0.5$ and $k_{g}=1$ are shown. Note that not only the slope but also the hysteresis (damping and friction) is scaled, i.e. the total torque is scaled with $k_{g}$ at all times.

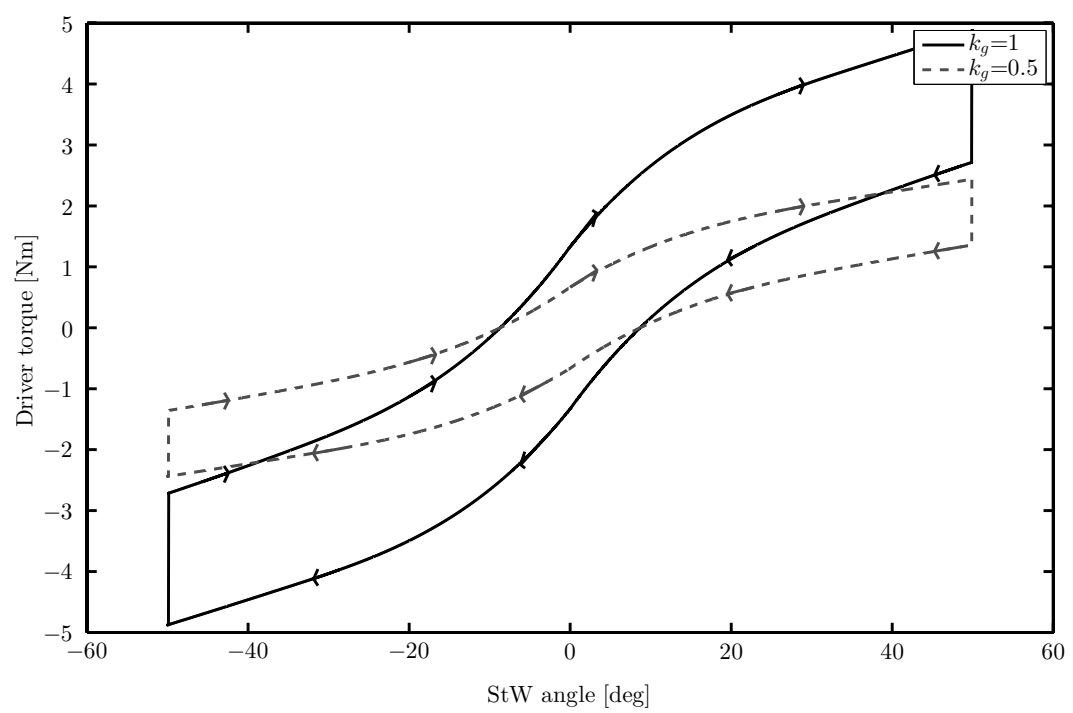

Figure 2 Steering wheel characteristic at speed $v_{x}=80 \mathrm{~km} / \mathrm{h}$ with scaling parameter $k_{g}$.

If the hypothesis of maintained driver force would hold true the scaling value $k_{g}$ should have a linear dependence on StW size, for each subject, i.e. each test driver. If on the other hand the $\mathrm{StW}$ torque was to be maintained $k_{g}$ should not change when the $\mathrm{StW}$ size varies. On top of the here described torque characteristics it was possible to superimpose more torque. This was used in the second part, described in next section.

\subsection{Part 2: Discontinuous Steering Characteristics}

A discontinuous steering column torque input was produced by a sudden vertical offset to the characteristics in Figure 2. In the experiment a square pulse was used as discontinuous torque input. The pulse lasted for one second and was $-3 \cdot k_{g} \mathrm{Nm}$. This gave obvious impact on the driver but was still considered as safe on the used test-track. The continuous characteristics were also scaled for part 2 as $k_{g}$ also effect relation (1). Again to scale complete torque applied on the StW. The rise time of the steering servo motor, from torque request to actual torque, was below $5 \mathrm{~ms}$. 


\subsection{Experiment}

To find out how force feedback should scale with StW size an experiment was set up where the previously described scaling method was used.

\section{Truck Specification}

A rigid Volvo FH16 truck was used. The axle arrangement was a single front axle, two rear axles whereof one driven. The rear most axle was lifted in all runs. Specifications are shown in Table 1. Three StWs were used, one Volvo FH $450 \mathrm{~mm}$ diameter (large), one Volvo V50 $390 \mathrm{~mm}$ diameter (medium) and a Sparco R333 $330 \mathrm{~mm}$ diameter (small). A quick release was installed to allow fast change of StW.

Table 1 Specification of Volvo FH16 truck used

\begin{tabular}{|c|c|c|c|}
\hline Property & Value & Unit & Description \\
\hline$L$ & 4.8 & $\mathrm{~m}$ & $\begin{array}{l}\text { Wheelbase, distance between front } \\
\text { and drive axle }\end{array}$ \\
\hline$F_{z, f}$ & 63044 & $\mathrm{~N}$ & Front axle vertical load \\
\hline$F_{z, d}$ & 72275 & $\mathrm{~N}$ & Drive axle vertical load \\
\hline$F_{z, t}$ & 0 & $\mathrm{~N}$ & Tag axle vertical load (lifted) \\
\hline$i_{s}$ & 23.2 & - & $\begin{array}{l}\text { Steering ratio, road wheel angle to StW } \\
\text { angle }\end{array}$ \\
\hline$I_{S t W}$ & {$[0.038,0.019,0.015]$} & $\mathrm{kg} \mathrm{m}^{2}$ & $\begin{array}{l}\text { StW inertia for large, medium and } \\
\text { small StW including StW and column } \\
\text { above power steering unit }\end{array}$ \\
\hline$r_{S t W}$ & {$[0.225,0.195,0.165]$} & $\mathrm{m}$ & $\begin{array}{l}\text { StW radius for large, medium and small } \\
\text { StW measured from centre to rim edge }\end{array}$ \\
\hline
\end{tabular}

The truck was equipped with Volvo Dynamic Steering which is a torque overlay electric steering servo mounted on-top of a hydraulic steering gear. The servo was controlled with a dSpace MicroAutoBox to realize scaling as described in section 2.1. In this way the value $k_{g}$ could be changed from a keyboard even at speed. More in detail, relation (1) was in fact implemented and verified fully. Including terms for aligning torque, damping, friction, steering wheel eccentricity and pulse. The only aspect not included in scaling was torque from $\mathrm{StW}$ inertia. The inertia of the $\mathrm{StW}$ together with steering column, $I_{S t W}$, was assumed small enough to be neglected.

\section{Track}

A handling track having a lot of bends and one straight section was used. The top speed was limited to $90 \mathrm{~km} / \mathrm{h}$. The track was approximately $3100 \mathrm{~m}$ long and $6.5 \mathrm{~m}$ wide with two lanes. Traffic was unidirectional and other vehicles, driving in the same direction, occurred. The track was dry and some short sections had normal disturbances such as dips, small crests or were rutted. The maximum lateral acceleration reached by most drivers was around $3 \mathrm{~m} / \mathrm{s}^{2}$. Drivers where advised not to drive slower than $45 \mathrm{~km} / \mathrm{h}$. One third of the track had an alternative route, with more bends. The subject were free to choose their preferred path, how they positioned and their speed as long as they stayed on road and within speed limits. 


\section{Subjects}

Totally 17 drivers participated; all holding truck driving license. From these 10 were full time test drivers and 7 development engineers. Two were female. The average driver was 39 years old, got the truck driving license in 1996 and drove $60000 \mathrm{~km}$ yearly in a truck.

\section{Set-up}

Each person was initially informed about purpose of the activity. This was followed by a warm up lap to get to know the truck and the track. Then the actual test was run.

Each subject ran with all three StWs. The order of the StWs was randomized. For each StW subjective tuning of continuous characteristics was first run, then followed by objective recording of discontinuous response. Here described:

Part 1: Tuning of Continuous Characteristics. The scaling parameter $k_{g}$ was used to adjust torque level when driving. The initial value of $k_{g}$ was randomized between 0.5 and 1.5, the subjects were told that the initial value was a random number and the actual value of $k_{g}$ was never shown to subjects. All of this was made to minimize cognitive bias (Fine, 2008), i.e. anticipation from subjects. The subjects were allowed to drive as many laps as needed to adjust the value of $k_{g}$ to find their optimal trade-off between comfort and handling. The subjects were instructed to change the value of $k_{g}$ by requesting either full, half or a quarter step up or down, where one full step corresponded to 0.1 in delta change of $k_{g}$. In this way the subjects were able to actively modify steering force level while driving and finally report their preferred level.

Part 2: Recording of Discontinuous Column Torque. The subjects were told to continue driving around the track, put both their hands on the $\mathrm{StW}$ and prepare for pulses to come (c.f. tensed mode in (Cole, 2012)). $k_{g}$ was set in random order to value 1.0, 0.85 or 0.7. These levels roughly correspond to $\mathrm{StW}$ diameter in relation to the large $\mathrm{StW}$ diameter. Note that changing $k_{g}$ effects both continuous characteristics and pulse size and that pulse was only given in one direction. An operator fired off pulses, unpredictable in time to subject, and all vehicle signals were recorded.

\section{Results and Discussion}

Here received results from the two parts are presented.

\subsection{Part 1: Continuous Properties}

The 17 subjects all decided on optimal scaling values of the driving torque characteristics. This was done for all the three StWs - resulting in 51 values of $k_{g}$, see Table 2. In Figure 3 the same data is shown but normalized with respect to the large $\mathrm{StW} k_{g}$ value per subject. The data is normalized since it is assumed that the preferred level is individual. Two lines are also included to visualise the hypothesis of maintained force and torque. This corresponds to slope 1 and 0 respectively.

By assuming normal distribution a paired t-test was used to show the likelihood of listed hypothesis, where three groups were used representing all StW sizes used. The hypothesis of maintained torque show $p<0.1 \%$ and is therefore rejected. The data is a lot more likely when assuming maintained force. Looking more in detail at the distribution of Figure 3 it can be seen that the number of data points are too few, as the variance is high, to guarantee 
Table 2 Final value of $k_{g}$ for all 17 subjects and StWs.

\begin{tabular}{llll}
\hline \hline Subject ID & Large & Medium & Small \\
\hline 1 & 0.9 & 0.8 & 0.55 \\
2 & 1 & 0.7 & 0.5 \\
3 & 0.9 & 0.85 & 0.55 \\
4 & 0.8 & 0.65 & 0.5 \\
5 & 0.9 & 0.75 & 0.65 \\
6 & 0.8 & 0.65 & 0.5 \\
7 & 0.85 & 0.8 & 0.55 \\
8 & 0.8 & 0.8 & 0.5 \\
9 & 0.9 & 0.55 & 0.5 \\
10 & 1 & 1 & 0.75 \\
11 & 1.05 & 0.8 & 0.7 \\
12 & 0.9 & 0.6 & 0.7 \\
13 & 1.05 & 1 & 0.7 \\
14 & 1.15 & 1.05 & 0.8 \\
15 & 0.75 & 0.75 & 0.55 \\
16 & 0.7 & 0.6 & 0.55 \\
17 & 0.9 & 0.65 & 0.45 \\
\hline
\end{tabular}

a linear relation. There are other non-linear models that would fit as good or better. The linear model of maintained force level is therefore only suggested as a rule of thumb for continuous steering assistance scaling. I.e. can be used as a first good guess.

During the trials some drivers complained about too slow StW free response return when $k_{g}$ was low, which could suggest a hypothesis of maintained free response return rate. The steering wheel return acceleration is a consequence of aligning torque minus dissipative forces, i.e. what is actually scaled with $k_{g}$. The method for scaling total torque should therefore only be used as a rough rule of thumb. More precise tuning of e.g. damping would also be needed, in fact it can be shown that damping should scale as $k_{g}^{2}$ to maintain free response return rate. The method used for scaling, where complete torque was varied with only one parameter, could be extended to make more requirements fulfilled.

Figure 3 show a rather large variance amongst drivers. Some additional tests were carried out with some of the subjects after completion of the main section. By changing $\mathrm{StW}$ and again initializing $k_{g}$ to a random number the question was if the driver would replicate his/her previous selection of $k_{g}$. Most of the subjects were not able to reproduce their previous value. In fact some drivers were obviously more sensitive than others. Some needed several laps to feel a difference when $k_{g}$ changed and some felt it instantaneously. This showed that most of the variance in Figure 3 comes from resolution ability amongst subjects. The Just Noticeable Difference, JND, describe the minimum difference required between two stimuli before a human can notice the difference between them. For the arm joints the JND of sensing force is around $7 \%$ and $2 \mathrm{deg}$ for angle (Tan et al., 1994). Assuming that the subjects compared steering stiffness, in terms of force per StW angle, at $40 \mathrm{deg}$ the JND for steering stiffness becomes $8.6 \%$, calculated with Taylor expansion of error propagation. This could explain a large part of the variance in Figure 3. Subjects were not able to discriminate between settings when $k_{g}$ changed by less than the JND. Whether a driver perceive force or torque would here be irrelevant, since the relative JND would be the same. Furthermore whether a driver perceive e.g. lateral acceleration rather than $\mathrm{StW}$ angle 
is left for future work. For this matter it could only add to the JND value, since sensing of force is here the dominant term. It should also be pointed out that the subjects had to keep the truck on the road, contributing with more uncertainty.

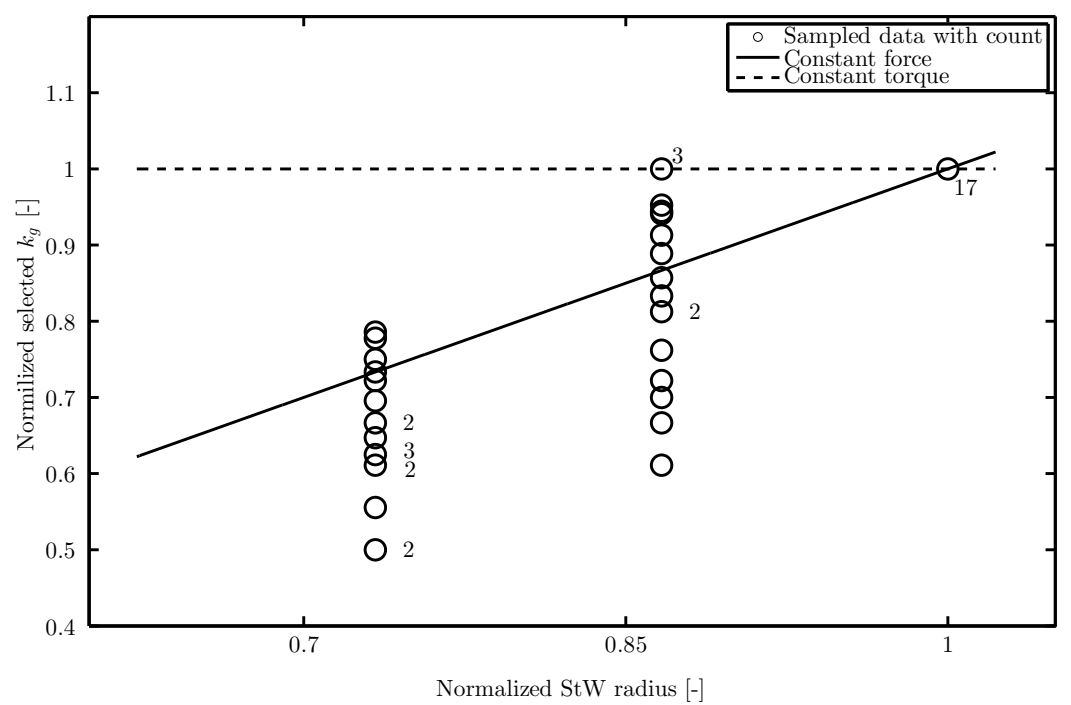

Figure 3 Result from test of steering assistance continuous properties. $k_{g}$ values are divided with $k_{g}$ from large $\mathrm{StW}$ per subject. $r_{S t W}$ is divided with $r_{S t W}$ from large $\mathrm{StW}$. E.g. all data-points for the large $\mathrm{StW}$ will lie in point $(1,1)$. Numbers are used to denote multiple occurrence of data-point. Also included is two lines, one with slope 1 and one with slope 0 , these are constrained to run through point $(1,1)$. The two lines correspond to constant force and constant torque respectively.

In the actual implementation of (1) it was assumed that torque from StW and column inertia could be neglected. A recording along the track is shown in Figure 4. It also includes an estimate of maximum error that is induced when neglecting scaling of inertia. The estimate is derived from $\mathrm{StW}$ angular acceleration times rotational inertia. As can be seen inertia is a lot smaller than other terms.

Used test procedure was set up to avoid bias in results from subject anticipation. Figure 5 show how initial value of $k_{g}$ effected the final value selected by the subjects. As can be seen the correlation is very low, which suggests that anticipation from subjects is low. After the continuous tuning activity all drivers were exposed to several pulses, as described in Section 2.3, this is the topic of the next section.

\subsection{Part 2: Discontinuous Properties}

In total 1080 pulses were recorded. Given two conditions, that speed has to be above 50 $\mathrm{km} / \mathrm{h}$ and that the magnitude of the StW angular rate has to be below $0.2 \mathrm{rad} / \mathrm{s}$ prior to pulse, 858 remain. Figure 6 show what effect that the pulses had on the StW angle $0.25 \mathrm{~s}$ after start of pulse. This was the point in time when the highest average deviation was seen. The shape of the distribution is not a normal distribution as seen. It is not symmetrical around the mean 


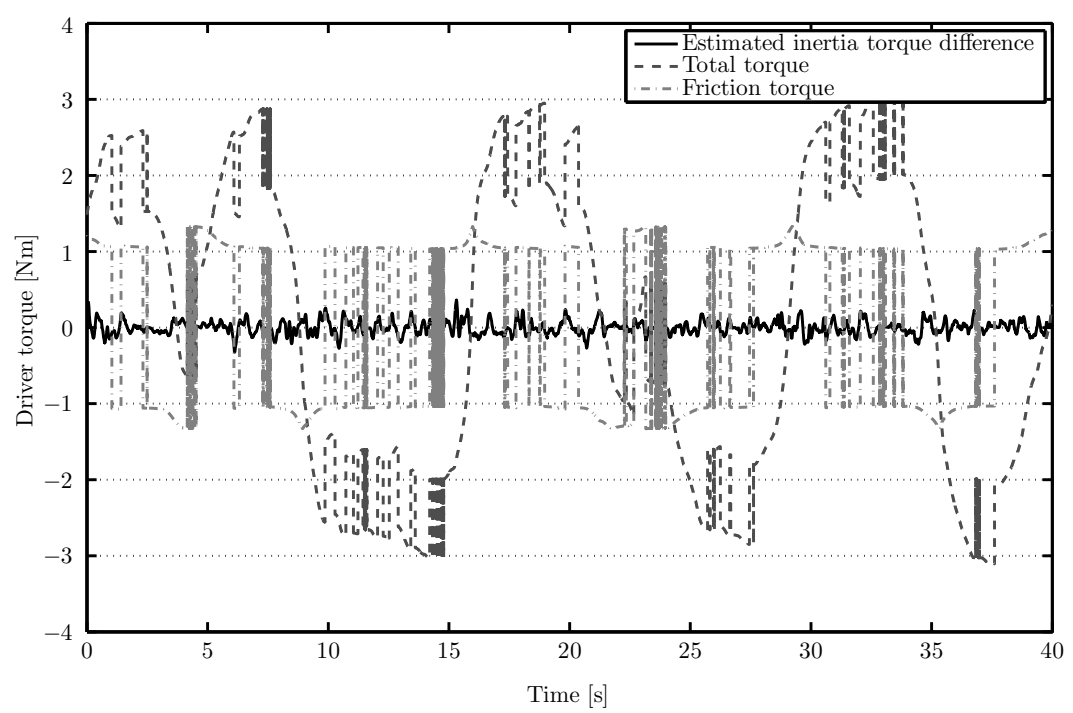

Figure 4 One measurement of total driver torque and friction along the track together with an estimate of error in torque arising when neglecting scaling of inertia.

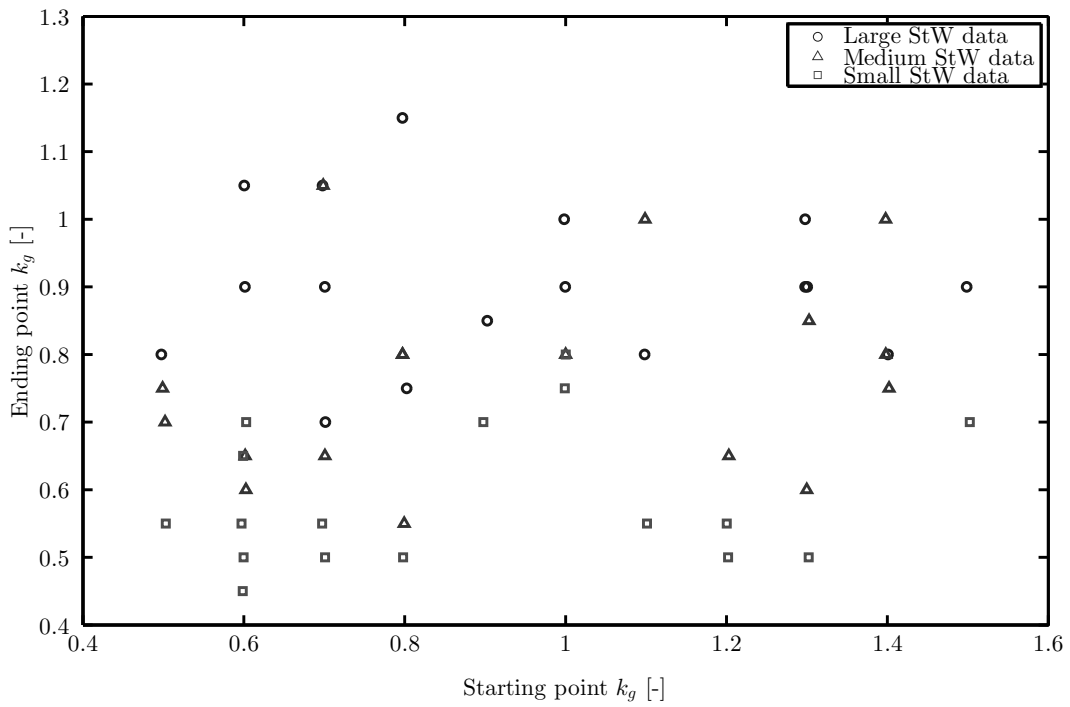

Figure 5 Final value of $k_{g}$ shown together with the randomized initial value. The correlation is very low. 
value and has a long tail on the left side. In total $88 \%$ responded in the same direction as the added torque. Possible reasons for the non-symmetrical shape are varying tension level of subjects, StW motion prior to the pulse and of course that there is different pulse sizes and StWs involved. However there are $12 \%$ of the pulses that even lead to a response opposite to the applied pulse. This indicate that the a priori uncertainty of a response is very high.

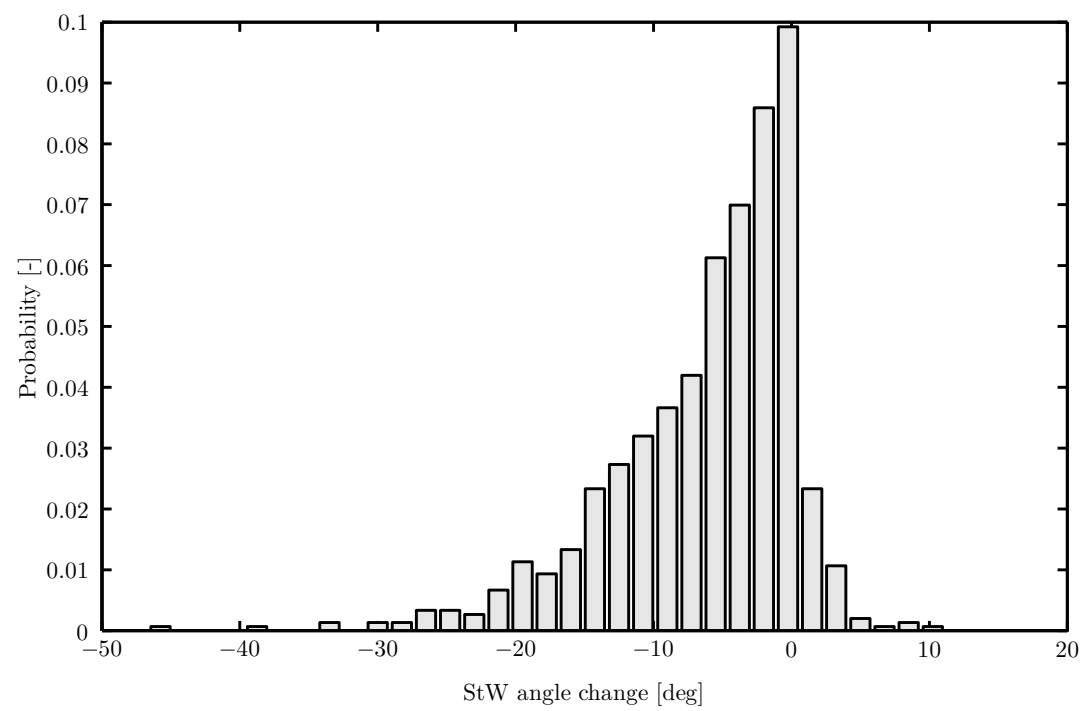

Figure 6 Sampled probability density function of all the 858 pulses. The variable shown is StW angle delta change $0.25 \mathrm{~s}$ from the start of the pulse.

The 858 pulses can be divided into nine classes, three $\mathrm{StWs}$ times three different values of $k_{g}$. Again mentioned, the pulse size was $-3 \cdot k_{g} \mathrm{Nm}$. For these histograms are shown in figure 7. Of special interest are the histograms on the diagonal, StW large $k_{g}=1, \mathrm{StW}$ medium $k_{g}=0.85$ and $\mathrm{StW}$ small $k_{g}=0.7$. These are close to be tuned as suggested in the rough rule of thumb presented in Section 3.1 - namely scaling of driver torque linearly with StW size to maintain force. I.e. the continuous characteristics are tuned to maintain driver force along the diagonal.

Can it also be said that a pulse should be scaled linearly with StW size to preserve driver angular response? To answer this question two more figures should be considered, Figure 8 and 9. These show how the mean delta change move as time passes. In Figure 8 the pulse is equal for all the StWs. In Figure 9 the pulse is scaled with StW size. The mean delta change is almost the same for all StWs, in the latter case, which is not so in the former case. This supports the hypothesis to scale discontinuous steering torque with StW size. The uncertainty is however big as seen from pooled standard deviation which is also included in both figures.

The inertia of the three StWs was not the same. Figure 10 show that the difference in torque, for the driver, cannot be fully neglected since the pulse itself is of order $3 \mathrm{Nm}$ and the error up to $0.5 \mathrm{Nm}$. However inertia conserves energy and the torque absorbed initially will be released when the motion is decelerated. This would result in a delay of the peak 

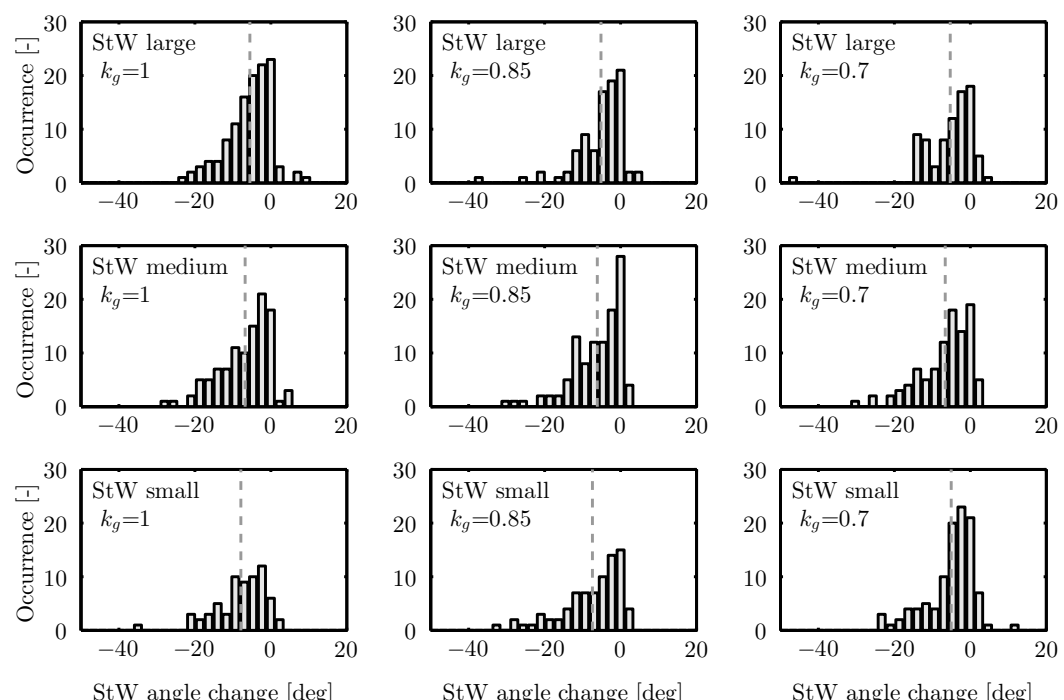

Figure 7 Histogram of StW angle delta change $0.25 \mathrm{~s}$ from the start of the pulse. Mean value of samples is shown with a vertical line. The y-axis show number of times that the delta change was observed.
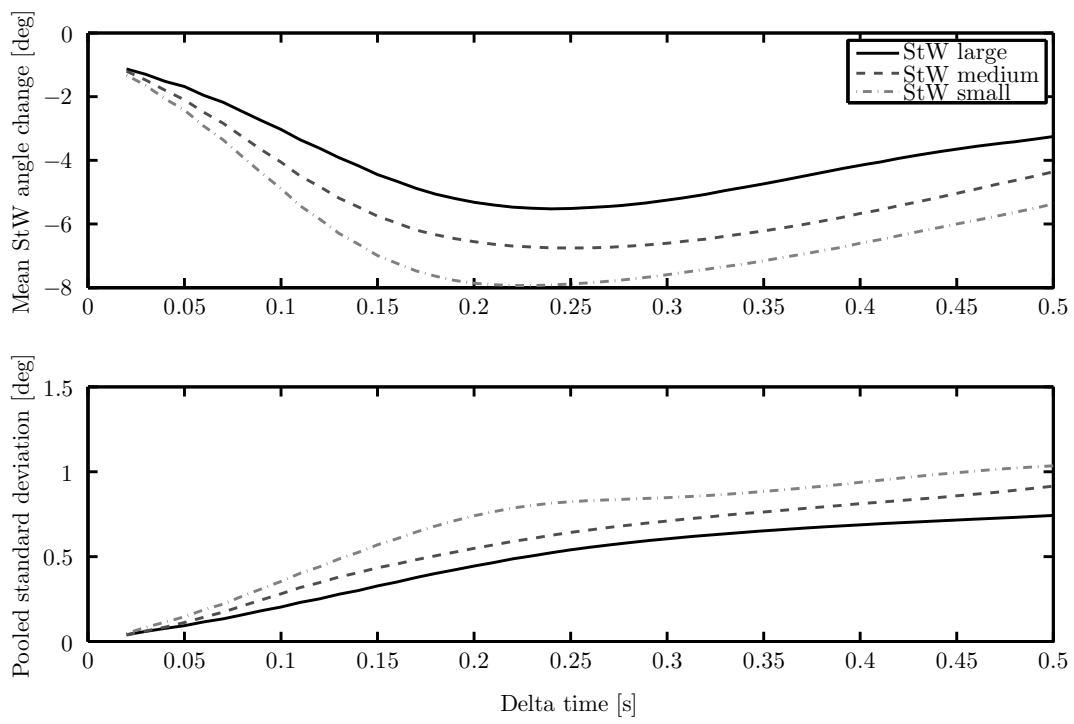

Figure 8 Mean StW angle delta change from start of pulse when $k_{g}=1$. Also pooled standard deviation is seen to grow as time moves. 

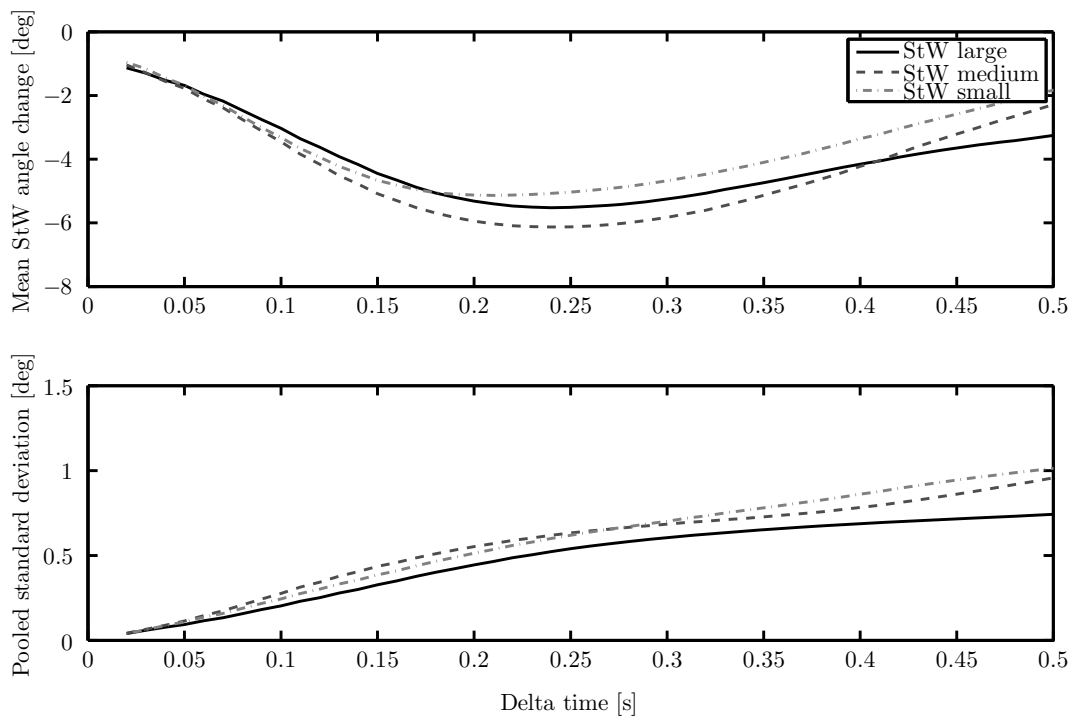

Figure 9 Mean StW angle delta change from start of pulse. For StW large $k_{g}=1$, medium $k_{g}=0.85$, small $k_{g}=0.7$. Also pooled standard deviation is seen to grow as time moves.

value in Figure 8 when the StW has higher inertia. Only small such tendencies can be seen. The error induced from inertia is therefore assumed to be of secondary effect.
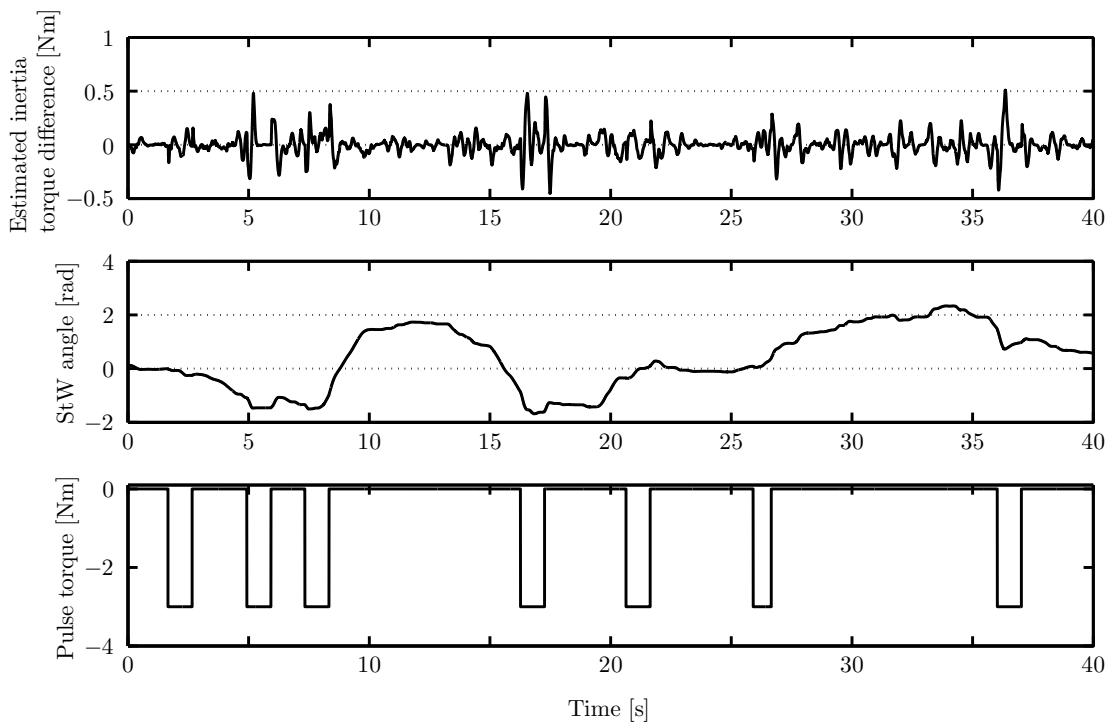

Figure 10 Maximum difference in torque from inertia, StW angle and pulse torque over time from a recording. 
The Influence of Steering Wheel Size when Tuning Power Assistance

\section{Conclusions}

An experiment has been set up, on a test-track, to show how continuous steering torque should depend on $\mathrm{StW}$ size; this to maintain a subjective balance between handling and comfort. A method was developed and implemented to scale complete torque felt by the driver at speed in a truck. 17 subjects tuned torque level for three different StWs, of different size. Initial scaling was random and not shown to the subjects. Also the order of the StWs was varied to avoid bias from anticipation. Results indicate that driver torque feedback should be scaled when StW size changes. A rule of thumb is to use linear scaling of total torque to accomplish maintained driver force level, which is in accordance with (Newberry et al., 2007). Further adjustment of damping, friction etc. might be needed to realise conservation of steering wheel free response return rate. The subjects had some problems to be consistent in their subjective judgement. The human resolution was identified as the main reason for this. Further studies on tactile resolution of steering forces are therefore suggested.

Also analysed was how discontinuous steering properties should depend on StW size to maintain the same angular driver response. I.e. how a torque pulse should scale with $\mathrm{StW}$ size to have the same effect on steering wheel angle, thereby vehicle lateral motion. The same rule of thumb as for continuous properties was shown to work, namely maintain force feedback level. The StW angle delta change response was seen to vary a lot. The distribution was not symmetrical and did thus not follow a normal distribution. In (Cole, 2012; Pick and Cole, 2007) the cases tensed and relaxed arms differ widely in response. The large variance here observed could therefore be a consequence of tension level. Other possible factors are driver arm inertia, strength and if the driver is entering/leaving a turn. By monitoring driver state it could be possible to reduce the uncertainty in repose (Abbink et al., 2011). This would be useful when designing steering support functionality and therefore recommended for future studies.

The study was conducted with fixed wheelbase, fixed understeer properties and fixed steering gear ratio. These are other possible reasons for having different power assistance level when varied.

\section{References}

Abbink, D.A., Mulder, M. and van Paassen, M.M. (2011) 'Measurements of muscle use during steering wheel manipulation', 2011 IEEE International Conference on Systems, Man, and Cybernetics, IEEE, Anchorage, pp.1652-1657.

Anand, S., Terken, J. and Hogema, J. (2011) 'Individual differences in preferred steering effort for steer-by-wire systems', Proceedings of the 3rd International Conference on Automotive User Interfaces and Interactive Vehicular Applications, ACM, New York, pp.55-62.

Barthenheier, T. and Winner, H. (2003) Das persönliche Lenkgefühl, fahrwerk.tech, Technische Universität Darmstadt, München, pp.1-17.

Ciarla, V., Cahouet, V., Canudas de Wit, C. and Quaine, F. (2012) 'Genesis of booster curves in electric power assistance steering systems', 2012 15th International IEEE Conference on Intelligent Transportation Systems, number Itsc, IEEE, Anchorage, pp.1345-1350.

Cole, D.J. (2012) 'A path-following driver - vehicle model with neuromuscular dynamics , including measured and simulated responses to a step in steering angle overlay', Vehicle System Dynamics: International Journal of Vehicle Mechanics and Mobility, Vol. 50, No. 4, pp.573-596.

Fine, C. (2008) A Mind of Its Own: How Your Brain Distorts and Deceives, 1st ed., WW Norton and Company. 
Howe, H.R. (1956) 'Mr Power steerings ship comes in', Popular Science, pp.161-164, 270.

Heissing, B. and Ersoy, M. (2010) Chassis Handbook (ATZ/MTZ-Fachbuch), 2011 ed., Vieweg und Teubner Verlag.

Kim, N. and Cole, D.J. (2011) 'A model of driver steering control incorporating the driver's sensing of steering torque', Vehicle System Dynamics, Vol. 49, No. 10, pp.1575-1596.

Newberry, A.C., Griffin, M.J. and Dowson, M. (2007) 'Driver perception of steering feel', Proceedings of the Institution of Mechanical Engineers, Part D: Journal of Automobile Engineering, Vol. 221, No. 4, pp.405-415.

Pfeffer, P.E. (2006) Interaction of Vehicle and Steering System Regarding On-Centre Handling, $\mathrm{PhD}$ Thesis, University of Bath.

Pick, a.J. and Cole, D.J. (2007) 'Dynamic properties of a driver's arms holding a steering wheel', Proceedings of the Institution of Mechanical Engineers, Part D: Journal of Automobile Engineering, Vol. 221, No. 12, pp.1475-1486.

Rossetter, E.J. (2003) A Potential Field Framework for Active Vehicle Lanekeeping Assistance, PhD Thesis, Stanford University.

Rösth, M. (2007) Hydraulic Power System Design in Road Vehicles, PhD Thesis, Linköping University.

Rothhämel, M. (2010) A Step Towards Implementation of Active Steering in Heavy Vehicles, Licentiate Thesis, Kungliga Tekniska Högskolan.

Salaani, M.K., Heydinger, G. J.G. and Grygier, P.A. (2004) 'Experimental steering feel performance measures', SAE Transactions, Vol. 113, No. 6, pp.680-683.

Tan, H.Z., Srinivasan, M.A., Eberman, B. and Cheng, B. (1994) 'Human factors for the design of force-reflecting haptic interfaces', Dynamic Systems and Control, Vol. 55, No. 1, pp.353-359.

UNECE (2005) Addendum 78: Regulation No. 79, Uniform Provisions Concerning the Approval of Vehicles with Regard to Steering Equipment, Revision 2.

Volvo Cars Corporation (2008) Press Information: Volvo Car Corporation Presents New Technologies to Avoid Collisions with Cars and Pedestrians.

Volvo-Trucks (2013) Press Information: Volvo Dynamic Steering, a Breakthrough for Effortless Steering without Strain or Pain.

Wheelskins-Inc (2009) Wheelskins Size Chart, [online] http://www.wheelskins.com/size_chart.php (Accessed 20 August 2013).

Yang, D. (2013) Vehicle Dynamics Control After Impacts in Multiple-Event Accidents, PhD Thesis, Chalmers University of Technology.

ZF-Lenksysteme (2012) Press Information: ZF Steering Systems Unveils Energy Saving High-Tech Solutions for the Commercial Vehicle Segment. 